\title{
THE GROWTH OF SCIENTIFIC KNOWLEDGE THROUGH THE SOCIAL COMPUTING NETWORKS
}

\author{
Baodong Liu \\ University of Utah, Salt Lake City, UT, USA
}

\begin{abstract}
While scholarly attention has been paid to the business impact of social computing, the effect of social computing on the growth of scientific knowledge itself demands further scholarly scrutiny. This paper analyzes the rapid development of open-source statistical learning and the increasingly dominant role played by social computing giants in the high-tech world exemplified by GitHub. Using the OLS regression diagnosis tools as a case study, this paper demonstrates how the growth of scientific knowledge is made possible in the causal chains of $\mathrm{R}$ statistical software development, R-package refinements and the application needs in the massive network of social computing.
\end{abstract}

\section{KEYWORDS}

Social Computing, Scientific Knowledge, R, GitHub

\section{INTRODUCTION}

The impact of social computing on socio economic outcomes has drawn increasing scholarly attention in the past decade. For example, social networking communities (e.g., FaceBook and YouTube) and business networking communities (e.g., Airbnb and Uber) have contributed to the unprecedented economic opportunities for individual users and firms alike (Fernado, et al, 2016). One underappreciated network community that deserves the same (if not more) scholarly scrutiny, however, is the scientific social computing networks that have provided the technological "knowhow" for social computing and the growth of the scientific knowledge in the first place. FaceBook, for example, has used Python for its infrastructure and production engineering. The Python community itself is not only composed of software engineers and data scientists, but also university faculty, clinical researchers, and industrial leaders.

In their systematic investigation into the causal chains involving how social computing eventually led to business benefits, Fernado et al (2016) indicated that technological developments in recent decade such as the cloud computing and web2.0 technology facilitated the rapid growth of the social computing networks. Fernado et al's original research, nonetheless, had a "narrow" goal in explaining the impact of these technological developments on the businesses and individual members of the social networks. It is imperative to raise the question about why there have been the technological revolutions to begin with. The early cooperation between leading universities was responsible primarily for the initial formation of the digital revolution. In particular, Stanford University, University of California-Berkeley, University of California-Los Angeles and University of Utah joined their computer networks on October 29, 1969 and formed the original internet. Moreover, scientists and engineers from industrial leaders (e.g., the Bell Lab and IBM) contributed to the developments of software and hardware technologies. But these early breakthroughs have been replaced by the new scientific network communities that provided the access to the detailed and nuanced technological knowhows at a speed never seen before.

One such leading scientific social computing network is GitHub. There were more than 40 million users as of the beginning of 2020 when about 190 million repositories were available on their web site which made GitHub the largest host of source codes in the world. GitHub was founded in 2008 and its main features such as bug tracking, task management, continuous integration made the company not only highly profitable (the company became a subsidiary of Microsoft in 2018), but also a leader in knowledge transmission, code development and scientific collaborations. Indeed, most of the exciting and highly influential research and development projects today are no longer controlled by one university or one firm only. Hundreds, if not 
thousands, of scientists and engineers from all over the world jointly contributed to the ever-evolving technological breakthroughs and growth of scientific knowledge. This paper uses the example of R-Package developments that significantly expanded and contributed to the accurate applications of the Ordinary Least Squares (OLS) Regression method (one of the oldest and most dominant data analysis tools) and visualization techniques to demonstrate how the scientific social computing networks such as R and GitHub contributed to the growth of scientific knowledge and the technological knowhows in the $21^{\text {st }}$ century.

\section{LITERATURE REVIEW}

Regression analysis has been one of the most commonly used statistical techniques ever since the first discovery of the least squares method by Carl Friedrich Gauss and Adrien-Marie Legendre by early $19^{\text {th }}$ century. The birth of personal computer industry in the 1980s and 1990s allowed the first wave of statistical software programs such as SAS and SPSS installed on PCs, and running an OLS model on a rectangular dataset with the dependent variable and independent variables specified rapidly empowered the users of statistical tools. However, most practitioners were taught only to empirically test their hypotheses, and they interpreted their OLS outputs without knowing that the fundamental assumptions of regression may have been violated. In doing so, the practitioners of regression tools often came to misleading or even totally wrong conclusions (Pedhazur, 1997). The failure to perform and/or report the necessary diagnoses of regression operations certainly was related to their lack of training in diagnostic techniques. Moreover, many early practitioners failed to do so because they did not have the tools to make diagnoses comprehensible. Osborne et al (2002) observed almost two decades ago, "few articles report having tested assumptions of the statistical tests they rely on for drawing their conclusions. This creates a situation where we have a rich literature in education and social science, but we are forced to call into question the validity of many of these results, conclusions, and assertions, as we have no idea whether the assumptions of the statistical tests were met."

The practice in writing up the results of OLS analyses without detecting whether the fundamental assumptions of OLS method were violated was widespread in the last century. Political scientist Christopher Achen vividly described the dominant role played by OLS, and yet the "political scientists" who published their major works based on OLS were also the ones who "pledge allegiance to a lord, and then throw off his livery" (Achen, 1982, p.11). At the time of publication of Achen's popular book on regression, the failure to perform diagnosis was a result of not only lack of training in the statistical theory but also the difficulty in learning the skill to perform diagnoses of OLS applications.

If the absence of performing OLS diagnosis naturally led to questions, and even concerns, regarding the real scientific value of the works published in the last century, researchers have almost no excuse in this century, especially in the past decade, for not performing OLS diagnosis. Scientists nowadays are much more empowered to perform the necessary diagnosis. In doing so, the published works in professional journals and books can bring a much higher level of scientific values, if OLS is used in the original discovery of these published works. There were many factors that contributed to the developments of tools critical to the quick, easy and intuitive process of OLS diagnosis. One major factor is the modern statistical computing technology that has become increasingly accessible, thanks to the popularity of powerful and free statistical software programs such as R. But the key technological breakthroughs were not simply a result of commercialized software developments represented by the earlier power-players such as SAS and SPSS. Even within the R project, the social computing network has been the key to the successful transition from a commercial-based to a user-based approach to growth of scientific knowledge.

Figure 1 shows how the new paradigm works and how the growth of knowledge is facilitated by a community of data scientists, software engineers, and users of application tools, and in other words, through the social-computing networks such as GitHub. The method of OLS regression has been dramatically improved in the last two decades so that when users apply this method the real growth of knowledge can take place (because the assumption of OLS are checked and the remedies are applied if any violation of OLS assumptions is detected). As shown in Figure 1, the mutual causal relationships start from the statistical language and software development called R. The software is freely available to anyone on the official R-project web site. Downloading and installing the R software allows users to apply many functionalities including OLS on their own datasets. However, to be able to diagnose whether the assumptions of OLS regression are violated due to misuse of this method on a certain dataset, the social computing networks, especially GitHub is critical. We demonstrate this model of growth of knowledge in more details below. 


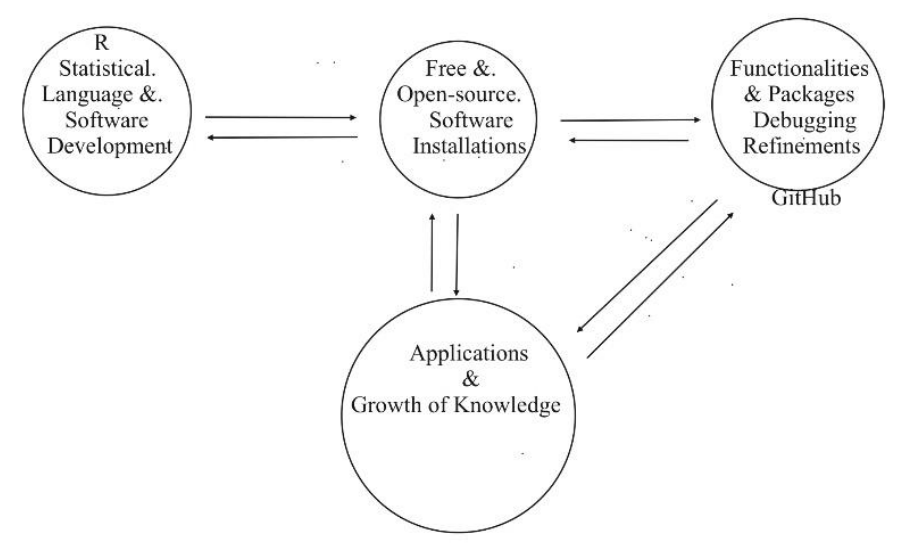

Figure 1. The Model of Growth of Knowledge through Scientific Social Computing Networks

\subsection{The OLS Assumptions}

"All statistical methods depend on assumptions" (Achen, 1982, p. 10). The basic idea of the so-called Least Squares approach is that the response variable is distributed conditionally based on one or a set of explanatory variables, and that the conditional distribution of response variable can be linearly explained by the minimization of sum of squared errors. Of course, the errors are the true distance between the observed response variable (y) and expected response variable given the certain value of an explanatory variable (x). This linear regression based on OLS approach allows the estimation of the regression coefficient vector, which includes an intercept. This method was made computationally easy in the early software programs such as SAS and SPSS.

After running the OLS model, a key question is whether the regression coefficients generated from the data are the true population parameters. SAS and SPSS, unfortunately, are not the programs that users can use to make OLS diagnosis, especially visualization tests we introduce below. It is important to note that the regression coefficients are only derived from an OLS model performed on a sample, which is supposedly drawn from the population. To make reasonable inferences about the population from a sample, it is necessary to follow the rule of central limit theorem. It is therefore essential to make the fundamental assumptions about how the data observed are generated in the first place. Here are the most important assumptions of OLS models.

I. Linearity: The expected values of response variable are a linear function of the explanatory variables.

II. Independency: The observations, or the units of analysis, must be made independent from each other.

III. Non-collinearity: The explanatory variables should have no strong correlation with each other.

IV. Constant Variance: The distributions of errors must be constant.

V. Normality of Variance: The errors are normally distributed.

VI. Measurement Error Free: The explanatory variables should not have measurement errors.

VII. The Model is Additive: The effect of one independent variable adds to the effect of another in the model to help explain the variation in the dependent variable.

\subsubsection{Diagnosis through Residual Visualization}

As the modern statistical computing technology became increasingly accessible, thanks to the popularity of powerful and open-source statistical software programs such as R, the operation of OLS is quick and easy with only one line of code after loading the data. The $\mathrm{R}$ linear function $(\mathrm{lm})$ will read the specified dependent variable $\left(\mathrm{y}_{\mathrm{i}}\right)$ and the matrix of independent variables $\left(\mathrm{X}_{\mathrm{i}}\right)$ regardless how the model is determined. This feature of OLS method is of course available in all statistical software programs such as SAS and SPSS. But R is different in that it allows new functionalities to be added as packages to its already powerful library system when the users initially installed the $\mathrm{R}$ software. The addition of new functions developed by scholars for diagnoses of OLS operations has helped professional data analysts to quickly and effectively examine their 
data and models for possible violations of assumptions. For example, John Fox (2002) developed the popular "car" R package which contains many visualization functions for OLS diagnosis. A typical R-package contains useful functions that users of $\mathrm{R}$ can call to apply to their own dataset so that the expected visual and statistical outputs can be generated. Furthermore, the refinements of R-packages can be accomplished after the source code is published and exchanged between and among users and developers in social computing networks, especially GitHub. This paper provides an overview of the most important diagnosis techniques and shows how to perform diagnoses of model assumptions through various $\mathrm{R}$ package which generates statistical tests as well as visual outputs automatically. The aim of this paper is to show how once the daunting task of performing basic visual and statistical diagnoses on OLS operations is made much more accomplishable in the era of free software environment for statistical computing. It is especially important to examine visualization tools.

With respect to OLS, residuals reveal a lot about the data structure. Residuals are actualizations of errors which are not observable. Errors should have a mean, a constant variance around the mean, and are normally distributed as well. One reason for the violation of constant variance assumption is that regression tends to produce small variance for those observations that have large leverages (i.e., the observations that have extreme $\mathrm{X}$ values). Liner model regression, by its nature, pulls the best fitting line/plane closer to those observations. The measurement of leverage for an observation is its hat-value, which measures the distance from the mean of $\mathrm{X}$. To take into consideration of the unequal contribution of observations given their hat-values, one can estimate the impact of residuals on regression results by studentizing the residuals (Hoaglin and Welsch, 1978), which is to use a t-distribution to assess not only the size of the residuals (as a numerator) but also the associated hat-value (as a denominator). We introduce seven residual plots that use mainly studentized residuals to perform various diagnoses of possible model-assumption violations. It is impossible to generate these plots in traditional statistical software programs such as SAS and SPSS. But GitHub has provided the social computing network that allowed countless R-packages being demanded, developed and refined. Table 1 summarizes the contents of these plots, and a running example of using the diaglm $\mathrm{R}$ package available on GitHub to generate these plots is also provided.

Table 1. Common Plots for Diagnosing OLS Assumption Violations

\begin{tabular}{lll}
\hline Plot Name & Horizontal Dimension & Vertical Dimension \\
\hline Influence Plot & Hat-values & studentized residuals \\
qqPlot & t or z values & studentized residuals \\
Marginal Model Plot & $\mathrm{X}_{\mathrm{i}}$ and fitted values & $\mathrm{Y}_{\mathrm{i}}$ \\
Density Plot & Studentized Residual & Estimated density \\
Residual Plot & Fitted Value & Studentized residuals \\
CrPlots & $\mathrm{X}_{\mathrm{j}}$ & partial residual for $\mathrm{X}_{\mathrm{j}}$ \\
avPlots & $\mathrm{X}_{\mathrm{i}}^{(1)}$ & $\mathrm{Y}_{\mathrm{i}}^{(1)}$ \\
\hline
\end{tabular}

Many functions have been written in the R environment to generate these plots and corresponding statistical tests. The most commonly used $\mathrm{R}$ package, car, is explained in details in the highly popular book written by Fox and Weisberg (2019). However, calling the specific $\mathrm{R}$ functions from car can be fairly time-consuming and intimidating without significant $\mathrm{R}$ programing experience. Thanks to social-computing networks, many OLS diagnosis R-packages are made available through GitHub. Typing the key search phrase on GitHub's powerful mobile app, "regression assumption", generated 238 repositories and 6208 issues on December 4, 2020. We introduce here the diaglm $\mathrm{R}$ package that provides the diagnosis plots and statistics automatically without calling the detailed $\mathrm{R}$ functions contained in car. The only condition to use this package is to successfully run an OLS model in the R environment, and the output has been generated without errors. To download the diaglm $\mathrm{R}$ package, type the following from the $\mathrm{R}$ command line (which starts with the $>$ sign):

$>$ install.packages('devtools')

$>$ devtools::install_github('bblpo/diaglm')

After installing the package, the user can start to use it to diagnose any linear models. Our running example uses the data originally contained in the diaglm R-package to follow examples of OLS models in a previously published article (Kittilson et al, 2011) that used the 2005 Citizen Involvement in Democracy survey data to examine whether virtual interactions enhanced citizen's social capital and political engagement in the United States. To have the access to the original data, load the diaglm $\mathrm{R}$ package by typing the following command: $>\operatorname{library}(\operatorname{diag} 1 \mathrm{~m})$ 
The above library $\mathrm{R}$ command will load all the functions in diaglm as well as the built-in dataset for replicating Kittilson et al (2011). To access the original dataset of Kittilson et al, type:

$>$ data(data)

The dataset now is called data in the global environment, and is ready for use. This article provides a diagnosis to the first model of Table 4 in Kittilson et al (2011, p. 639). We first run the OLS model as specified in the original article. We assign the name, toler, to the first model of Kittlson et al's Table 4. On the R command line, type the following OLS model according to the original article:

$>$ toler $<-\operatorname{lm}$ (tolerance_scale $\sim$ sg_participation+virtual_si+as.numeric(EDUC)+as.numeric(yrbrn)

+IVRACE2+IVRACE3+gndr+as.numeric(lrscale), data=data, weights $=$ NATWT)

After successfully running the above OLS model, the diaglm $\mathrm{R}$ package can be called to diagnose the possible violation of OLS assumptions:

$>$ diaglm(toler)

$\mathrm{R}$ should then display all the graphic outputs of your diagnoses, and save the statistical test results into a file called result.txt in your working directory. If the diaglm() command is successfully executed, the residual plots are automatically generated and saved in the working directory. Seven residual plots are listed in Table 1 which are available in the diaglm R-Package. We briefly introduce only the first two visual methods here.

\subsubsection{Influence Plot}

The existence of unusual and influential data may make the model an inaccurate reflection of the true relationship between the response variable and explanatory variables. Influence plot shows both leverage (the horizontal dimension) and studentized residuals. The size of the circles is proportional to Cook's D (Cook, 1977). Influence plot can be used along with qqPlot and avPlots (discussed below) to identify the observations which are unusual cases.
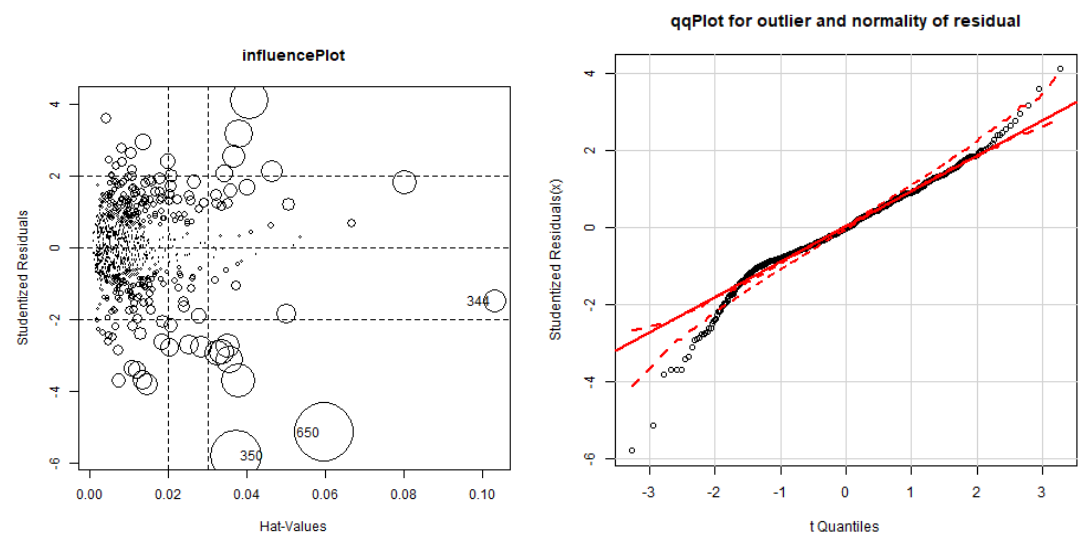

Figure 2. Influence Plot and qqPlot

The examination of unusual cases certainly leads to the question about whether or not it is necessary to remove such cases. While it has been suggested that removing observations "will distort the parameter estimations", Osborne (2017) argued that "it is defensible to remove cases that have a low probability of being part of the population" (p. 38, also see Osborne 2013). If the number of observations is small, removing these unusual cases can change the model estimations significantly. Thus, it is necessary to examine the data to find the reason for unusual cases in the first place before removing them from the dataset.

Figure 2 shows the Influence Plot on the left panel for the Kittilson et al (2011) model. Note that the 350th and 650th observations appear to be problematic as their studentized residuals are below -5, and their Cook's D values are the largest, while the $344^{\text {th }}$ observation has the largest leverage value (Hat-value greater than .1), which may also lead to biased model estimations. Removing these three observations, however, did not lead to the changes in statistical significance for the explanatory variables. Thus, there is no evidence that these unusual cases have caused serious problems for statistical interpretations for the Kittilson et al model. 


\subsection{3 qqPlot}

The qqPlot is the major visual check on the distribution of residuals. This plot allows users to find whether the residuals are distributed normally. If all the observations are within the $95 \%$ confidence envelop diagonally, then the residuals are normally distributed, and the overall regression model is a good fit. If the observations are outside the $95 \%$ confidence envelop, then we have situations of non-normally distributed residuals, which suggested that the model is not a good fit. Regarding the first model of Table 4 in Kittilson et al (2011), the qqPlot (see the right-side of Figure 1) revealed a positive skew of residuals, which suggests a need to transform the response variable

\section{DIAGNOSIS THROUGH STATISTICAL TESTS}

In addition to using residual plots to perform diagnoses on regression results, the diaglm $\mathrm{R}$ package also provides the statistical test results. It is also useful to perform these statistical tests to double check the conclusions drawn from diagnostic plots. The following tests are reported automatically by diaglm.

\subsection{The Outlier Test}

This test will list the observations whose studentized residuals failed to pass the certain cutoff value of Bonferonni $p$ (Dunn, 1958). The default is .05. These observations are outliers, which may cause problems on the error distributions.

\subsection{The DubinWatson Test}

The Dubin Watson test estimates the possibility of autocorrelations, which are the situations when the observations are not independent from each other (Durbin et al, 1950). Since it is a statistical test, one can look at its $\mathrm{p}$ value. If it is smaller than .05 , there is a good reason to conclude that the autocorrelation among observations is indeed serious.

\subsection{The Collinearity Test}

The most common statistical test to detect collinearity is the Variance Inflation Factor (VIF) test (see Fox, 2016). The critical value for the VIF estimates is 4 . If a certain $X$ variable has a larger than 4 VIF, this variable is highly correlated with other explanatory variable(s).

\subsection{The Global Test}

This test combines the statistics from global statistics, skewness of residuals, Kurtosis test which evaluates the normality of the residuals, link function, and heteroscedasticity test (Pearson 1929). This test can be used along with other graphic approaches illustrated above.

\section{DIAGNOSES AND SOLUTIONS}

The visual and statistical tools introduced above provide powerful and effective ways to examine whether the fundamental assumptions of OLS have been violated when certain hypotheses are stated and empirical data are used. But when a violation of OLS assumptions has been discovered, what are the feasible solutions to the violation problems? It is certainly not a good practice to abandon the whole dataset just because a certain assumption of OLS is violated. The development of R-package such as diaglm provides a further checklist for users of OLS when they see problems concerning residual distribution, non-linearity, autocorrelation, and lack of goodness of fit. The refinements of R-packages through social computing network GitHub allow the growth of scientific knowledge when empirical data in all scientific fields can be maximized for scientific use. 


\subsection{The Residual Distribution Problem}

When residual plots show that the distribution of studentized residuals is a clear violation of constant and normal error distribution principle of linear model, it is important to find why that is the case. Sometimes, it is caused by some unusual cases in the dataset. If that turns out to be the case revealed by plots such as Influence plot, delete those cases with very high values of Cook's D. If the deletion of these cases does help to improve the residual distribution, then it is a successful move. Note that it is also important to study why certain observations are unusual in the first place. Finding the reasons for existence of unusual cases often turns out to be the turning point of finding good model that allows researchers to explain their response variable. Sometimes, the model simply needs to exclude some irrelevant explanatory variables or add some critically important explanatory variables that are missing. Especially, when a model produces some systematic features for residual distribution, such as bimodal or multimodal distributions of residuals. In these cases, it is very likely that some categorical variables can be entered into the model to capture these patterns of errors. Of course, a good theory is needed to explain why some ignored variable(s) are important. Sometimes, the violation of residual distribution assumptions can be caused by the nature of the response variable. One may try to see whether the transformation of the response variable can help improve the fit of the model.

\subsection{The Violation of Linearity Assumption}

By transforming the response variable the nonlinear relationship problem, sometimes, may be solved. If the curvilinear relationship continues, and the CrPlot shows a clear a curvilinear relationship, then it is time to consider to transform the explanatory variable. First, identify whether the curvilinear relationship is monotone or non-monotone (Fox, 2016). Monotone curvilinear relationship means that change in the X value will lead to the consistently increases or decreases in $\mathrm{y}$, though the rate of change is different throughout the $\mathrm{X}$ values. If there is a change in the direction of relationship between the response variable and the given $\mathrm{X}$, for example, from positive to negative, then the curvilinear relationship becomes non-monotone. How much should one increase or decrease the ladder of powers? Should $\mathrm{X}$ be transformed to $\mathrm{X}^{2}$ or $\mathrm{X}^{3}$, for example, when the CrPlot shows a curve like the top-right one in the Tukey and Mosteller's chart? The diaglm R package run the transformX () function to find the best power transformation of X generated by Maximum Likelihood method through the transformX.nocontrol() function. Finally, when the curvilinear relationship between the response variable and a given $\mathrm{X}$ variable is not monotonic as shown by CrPlot, then run a polynomial regression.

\subsection{The Autocorrelation Problem}

If the autocorrelation happens due to the fact that units are correlated with themselves, it is useful to ask what is the cause. Sometimes, the units are "too close" to each other. For example, some units are from the same geographic unit (e.g., the same county or state) which contributed a common pattern observed from the data. In this case, users can perform a clustered regression, and take into account that observations are clustered. Hierarchical linear modeling (HLM) is especially promising to address the autocorrelation problem caused by hierarchical data structure. It is also possible that units are correlated due to the fact that the data are collected over time and the same units show different values simply because they experience changes over time. But still they are the same units. In this case, it is important to take into consideration of time. One may try time-series analysis. Sometimes, modeling can get much more complex if the nature of the data is time-series and cross-sectional. One will need special tools to address the autocorrelation issue in these complex situations.

\subsection{The Lack of Good Fit Problem}

It is possible that no matter what one attempts to do (such as using transformation of response variable, transforming explanatory variables, dropping the key unusual cases, taking auto-correlation issue into consideration), very little, if any, has been changed. The $\mathrm{R}^{2}$ is still small, the adjusted $\mathrm{R}^{2}$ shows no improvement, the residuals are still distributed very unusually, and none of explanatory variables come out statistically significant. Why? It is likely that some variables are measured very badly with very low validity; or the independence of observations cannot be sustained no matter how one wants to use tools such as clustered 
regression to remedy it. The right specification is the condition for accurate estimation of slope coefficients. The re-specification can be successfully implemented by adding or dropping one or more independent variables, which is still based on the "additive" model assumption. Very often, however, differentiating additive and conditional relationships is the key to the finding of a correct specification.

\section{CONCLUSION}

The rapid development of R-packages thanks to the powerful social computing network GitHub allows the rapid growth of scientific knowledge itself. This paper provides an overview of important diagnosis techniques of OLS model assumptions through the diaglm R packages which generate not only statistical tests but also visual outputs which are not available in traditional statistical software programs such as SAS and SPSS. Using a running example of the diagnosing an OLS model reported in a published article, this paper introduces seven residual plots and the major statistical tests concerning OLS model assumptions by using R packages which are freely available to any user, thanks to the social-computing network, GitHub. The paper further shows the way to deal with potential problems of model-assumption violations. The technique of transformation of the response and explanatory variables is also introduced. In short, this paper demonstrates that once the daunting task of performing basic visual and statistical diagnoses on OLS operations is made much more accomplishable in the era of free and open-source statistical software and the social-computing networks. It is also worth noting that users of GitHub can bring their application issues concerning any R-package any time to the social computing networks, and seek for helps in the community. In doing so, the growth of scientific knowledge indeed becomes a matter of helping each other through a village, rather than working on issues as a hermit.

\section{REFERENCES}

Achen, C., 1982. Interpreting and Using Regression. Sage Publication, Inc, Newsbury Park, USA.

Cook, R., 1977. Detection of Influential Observations in Linear Regression. Technometrics, Vol.19, pp.15-18.

Dunn, O., 1958. Estimation of the Means for Dependent Variables. Annals of Mathematical Statistics, Vol. 2, No. 4, pp.1095-1111.

Dubin, J. et al, 1950. Testing for Serial Correlation in Least Squares Regression II. Biometrika, Vol. 37, pp.409-28.

Fernando, M., et al, 2016. Enhancing Business Outcomes through Social Computing. LADS International Journal of WWW/Internet. Vol. 14, No. 2, pp.91-108.

Fox, J. 2016. Applied Regression Analysis and Generalized Linear Models. Sage, Los Angeles, USA.

Fox, J. 2002. An R and S-Plus Companion to Applied Regression. Sage, Los Angeles, USA.

Fox, J. et al, 2019. An R Companion to Applied Regression. Sage, Los Angeles, USA.

Hoaglin, D. et al, 1978. The Hat Matrix in Regression and ANOVA. American Statistician, Vol. 32, pp.17-22.

Kittilson, M. et al, 2011. Virtual Civil Society: The New Frontier of Social Capital? Political Behavior, Vol. 33, No. 4, pp.625-644.

Mosteller, F. et al, 1977. Data Analysis and Regression. Addison-Wesley, Reading, USA.

Osborne, J., 2013. Best Practices in Data Cleaning: A Complete Guide to Everything You Need to Do Before and After Collecting Your Data. Sage, Thousand Oaks, USA.

Osborne, J. et al, 2002. "Four Assumptions Of Multiple Regression That Researchers Should Always Test." Practical Assessment, Research \& Evaluation Vol. 8, No. 2.

Pedhazur, E., 1997. Multiple Regression in Behavioral Research. Harcourt Brace, Orlando, USA.

Pearson, K., 1929. "Editorial Note to 'Inequalities for Moments of Frequency Functions and for Various Statistical Constants"”. Biometrika Vol. 21, No. 1-4, pp.361-75. 